\title{
IM-412 inhibits the invasion of human breast carcinoma cells by blocking FGFR-mediated signaling
}

\author{
SEUNG-YOUN JUNG ${ }^{1}$, JAE YOUN YI ${ }^{2}$, MI-HYOUNG KIM ${ }^{1}$, KYUNG-HEE SONG ${ }^{1}$, SEONG-MOOK KANG ${ }^{1}$, \\ JIYEON AHN $^{1}$, SANG-GU HWANG ${ }^{1}$, KY-YOUB NAM ${ }^{3}$ and JIE-YOUNG SONG ${ }^{1}$
}

\author{
${ }^{1}$ Division of Radiation Cancer Research, ${ }^{2}$ Division of Radiation Effect, Korea Institute of Radiological and Medical Science; \\ ${ }^{3}$ Center for Development and Commercialization of Anti-Cancer Therapeutics, Asan Medical Center, Seoul, Republic of Korea
}

Received June 3, 2015; Accepted July 29, 2015

DOI: $10.3892 /$ or.2015.4249

\begin{abstract}
Triple-negative breast cancer (TNBC) is an aggressive cancer with a poor prognosis due to its epithelial-to-mesenchymal transition (EMT) phenotype. Cancer patients often experience several detrimental effects of cancer treatment, such as chemoresistance, radioresistance and the maintenance of cancer stem cells due to EMT. Thus, EMT signaling is considered to be a valuable therapeutic target for cancer treatment, and its inhibition is being attempted as a new treatment option for TNBC patients. Previously, we showed that 3-(2-chlorobenzyl)-1,7-dimethyl-1H-imidazo[2,1f]purine-2,4(3H,8H)-dione (IM-412) inhibits transforming growth factor- $\beta$ (TGF- $\beta$ )-induced differentiation of human lung fibroblasts through both Smad-dependent and -independent pathways. In the present study, we examined the inhibitory effect of IM-412 on EMT pathways and invasiveness in TNBC cells since the TGF- $\beta$ signaling pathway is a typical signaling pathway that functions in EMT. IM-412 not only potently suppressed the migration and invasion of MDA-MB-231 cells, but also lowered the expression of mesenchymal markers and EMT-activating transcription factors in these cells. IM-412 inhibited the activation of several signaling proteins, including Smad2/Smad3, p38MAPK, Akt and JNK, and it also attenuated the phosphorylation of FGFR1 and FGFR3. Collectively, our findings suggest that the synthetic compound IM-412 suppressed the EMT process in MDA-MB-231 cells and thereby effectively inhibited the migration and invasion of these cancer cells. Thus, IM-412 could serve as a novel therapeutic agent for malignant cancers.
\end{abstract}

Correspondence to: Professor Jie-Young Song, Division of Radiation Cancer Research, Korea Institute of Radiological and Medical Sciences, 215-4 Gongneung-dong, Nowon-gu, Seoul 139-706, Republic of Korea

E-mail: immu@kirams.re.kr

Key words: IM-412, triple-negative breast cancer, epithelial-tomesenchymal transition, migration, invasion, FGFR

\section{Introduction}

A common feature of progressive cancers is metastasis to other tissues. For migration out from their primary site, cancer cells transform into cells that exhibit mesenchymal phenotypes, and this transition is called epithelial-to-mesenchymal transition (EMT). Physiologically, EMT has been associated with development, wound healing, inflammation, fibrosis and cancer-cell invasion and migration (1-3). In numerous cancers, EMT presents several disadvantages to patients, such as radioresistance, chemoresistance and maintenance of cancer stem cells (4-6). Thus, EMT signaling is considered to be a favorable therapeutic target for cancer treatment $(7,8)$.

EMT is induced by diverse secreted molecules such as fibroblast growth factor (FGF), hepatocyte growth factor, epidermal growth factor (EGF), platelet-derived growth factor and transforming growth factor $-\beta$ (TGF- $\beta$ ), and among these, TGF- $\beta$ is well characterized as a key inducer of EMT in various types of cancers $(1,6)$. The TGF- $\beta$ signaling pathway involves the following steps: TGF- $\beta$ binds to TGF- $\beta$ receptor II (T $\beta$ RII), which then heterodimerizes with TGF- $\beta$ receptor I (T $\beta$ RI). Next, the TGF- $\beta$ receptors are phosphorylated and they activate downstream signaling pathways, including the canonical Smad signaling pathway and the non-Smad signaling pathway. Subsequently, various EMT markers and associated molecules are transcriptionally regulated by EMT-regulating transcription factors such as SNAI1 (snail family zinc finger 1; SNAIL) and SNAI2 (snail family zinc finger 2; SLUG), zinc finger E-box-binding homeobox 1 and 2 (ZEB1 and ZEB2) and twist family bHLH transcription factor 1 (TWIST1) $(1-3,9)$.

FGF receptors (FGFRs) constitute another family of receptors associated with several cellular processes in cancer, including metastasis, stemness, proliferation, anti-apoptosis, drug resistance and angiogenesis $(10,11)$; thus, FGFRs have recently been considered as a potential therapeutic target for cancer patients (10-12). Four major FGFRs are expressed by cells, and among these, FGFR1 is highly involved in metastasis and FGFR3 contributes to cancer-cell metastasis. Moreover, the FGFR4 SNP variant Gly388Arg was shown to be associated with metastasis, while FGFR2 acts as an antagonist and suppresses the EMT process (13-15).

Previously, we demonstrated that 3-(2-chlorobenzyl)-1,7-dimethyl-1H-imidazo[2,1-f]purine-2,4(3H,8H)-dione (IM-412) 
inhibits TGF- $\beta$-induced fibroblast differentiation through both Smad-dependent and -independent pathways in human lung fibroblasts (16). Since IM-412 exerted an anti-fibrotic effect by inhibiting the TGF- $\beta$ signaling pathway, in the present study, we investigated whether IM-412 also affects EMT signaling and the invasiveness of cancer cells.

\section{Materials and methods}

Materials. We obtained primary antibodies against the following proteins from commercial sources: p-Smad2 (Ser245/250/255), p-Smad2 (Ser465/467), Smad2, p-Smad3, Smad3, p-p38MAPK, p-Akt, Akt, p-JNK, JNK, p-ERK, p-c-Jun and c-Jun (Cell Signaling Technology, Danvers, MA, USA); E-cadherin, fibronectin, collagen type 1 A2, p38MAPK, ERK and T $\beta$ RI (Santa Cruz Biotechnology, Santa Cruz, CA, USA); N-cadherin, SNAIL, SLUG and ZEB1 (BD Biosciences, Palo Alto, CA, USA); vimentin and $\alpha$-SMA were obtained from Sigma (St. Louis, MO, USA); p-FGFR3, FGFR3 and T $\beta$ RII (Abcam, Cambridge, MA, USA); and GAPDH (AbFrontier, Seoul, Korea). Horseradish peroxidase (HRP)-conjugated secondary antibodies were purchased from Thermo (Pittsburgh, PA, USA). For use in the in vitro invasion assay, Transwells were purchased from Corning and Matrigel was purchased from BD Biosciences. IM-412 $\left(\mathrm{C}_{16} \mathrm{H}_{14} \mathrm{ClN}_{5} \mathrm{O}_{2}\right.$, MW: 344; ID 9073517) was isolated from a chemical library of screening compounds and was purchased from ChemBridge Corporation (San Diego, CA, USA).

Cell culture. The human breast cancer cell line MDA-MB-231 was purchased from the American Type Culture Collection (ATCC; Manassas, VA, USA). The cells were maintained at $37^{\circ} \mathrm{C}$ in RPMI-1640 supplemented with $10 \%$ fetal bovine serum (Gibco-BRL, Grand Island, NY, USA), $100 \mathrm{U} / \mathrm{ml}$ penicillin and $100 \mu \mathrm{g} / \mathrm{ml}$ streptomycin in a humidified atmosphere containing $5 \% \mathrm{CO}_{2}$.

MTT assay. Cell viability was assessed using the 3-[4,5-dimethylthiazol-2-yl]-2,5-diphenyltetrazolium bromide (MTT) assay (Sigma). MDA-MB-231 cells were plated at a density of $8 \times 10^{3}$ cells/well into 24-well plates (SPL, Gyeonggi, Korea), and the seeded cells were stabilized for $24 \mathrm{~h}$, and were subsequently treated with each indicated dose of chemicals. MTT was directly added to each well at a final concentration of $0.5 \mathrm{mg} / \mathrm{ml}$, and after $4 \mathrm{~h}$, the medium was removed, the formazan crystals in MDA-MB-231 cells were dissolved in dimethylsulfoxide and the absorbance of the formazan solution was measured using a microplate reader (Multiskan EX; Thermo LabSystems, Waltham, MA, USA) equipped with a $540-\mathrm{nm}$ filter. Each sample was assayed in triplicate, and the experiment was repeated thrice.

In vitro wound migration assay. Cells were seeded into 6 -well plates $(\mathrm{SPL})$ at $90 \%$ confluency $\left(5 \times 10^{5}\right.$ cells/well) and incubated overnight. On the next day, the culture medium was aspirated, and the cells were scratched using a $200-\mu 1$ pipette tip. After wounding, the cultures were washed with phosphate-buffered saline (PBS) and treated with IM-412. The cells were allowed to migrate for $24 \mathrm{~h}$ and were subsequently stained with crystal violet. Migration patterns were examined under a microscope and photographed at a magnification of $\mathrm{x} 40$.

In vitro Transwell invasion assay. The invasiveness of the cultured cells was determined using a 24-well Transwell system (pore size, $8-\mu \mathrm{m}$ ) (Corning Incorporated, Corning, NY, USA). The upper side of the Transwell membrane was coated with $1 \mathrm{mg} / \mathrm{ml}$ Matrigel (in $10 \mu \mathrm{l}$ ) and then cells were seeded into the upper chamber at a density of $5 \times 10^{4}$ cells in $100 \mu \mathrm{l}$ of media; the complete medium was added to the lower chamber. Cells were incubated for $24 \mathrm{~h}$ at $37^{\circ} \mathrm{C}$ in $5 \% \mathrm{CO}_{2}$, and then the cells that had not penetrated the filter were wiped away using cotton swabs; the cells that had invaded the lower surface of the filter were fixed with methanol, stained with crystal violet, examined under a microscope, and photographed at a magnification of $x 40$. The numbers of invaded cells were determined by counting the cells in 5 randomly selected regions.

Western blot analysis. Cells were lysed with RIPA buffer (50 mM Tris-Cl, pH 7.4, 1\% NP-40, $150 \mathrm{mM} \mathrm{NaCl,} 1 \mathrm{mM}$ EDTA) supplemented with protease inhibitors (1 $\mathrm{mM}$ phenylmethylsulfonyl fluoride, $1 \mu \mathrm{g} / \mathrm{ml}$ aprotinin and $1 \mu \mathrm{g} / \mathrm{ml} \mathrm{leupeptin)}$ and phosphatase inhibitors ( $1 \mathrm{mM} \mathrm{Na}_{3} \mathrm{VO}_{4}$ and $1 \mathrm{mM} \mathrm{NaF}$ ). Proteins in the whole-cell lysates were separated on $8-15 \%$ SDS-polyacrylamide gels and transferred to nitrocellulose membranes (Bio-Rad, Hercules, CA, USA). The membranes were blocked $(1 \mathrm{~h})$ with $5 \%$ skim milk in tris-buffered saline containing $0.1 \%$ Tween-20 and then probed with primary antibodies (overnight, $4^{\circ} \mathrm{C}$ ). After multiple washes, the membranes were incubated with HRP-conjugated secondary antibodies, and then the immunoreactive bands were detected using enhanced chemiluminescence reagents according to the manufacturer's recommendations (GE Healthcare; Little Chalfont, UK). The experiments were repeated at least thrice.

Immunocytochemical staining. MDA-MB-231 cells $\left(5 \times 10^{5}\right.$ cells) were grown on glass coverslips, stabilized for $24 \mathrm{~h}$ and then treated for $24 \mathrm{~h}$ with the indicated doses of IM-412. Next, the cells were washed with Hank's balanced salt solution (HBSS), fixed with $3.7 \%$ formaldehyde in HBSS for $10 \mathrm{~min}$ at room temperature, and then washed and permeabilized with $0.1 \%$ Triton X-100 in HBSS for $15 \mathrm{~min}$ at room temperature. Mouse $\alpha$-SMA antibody (1:500) was used as the primary antibody and it was detected using an Alexa 546-conjugated secondary antibody. Nuclei were counterstained with $300 \mathrm{ng} / \mathrm{ml}$ 4',6-diamidino-2-phenylindole (DAPI; Invitrogen, Carlsbad, CA, USA). Coverslips were mounted on glass slide with Gel/Mount ${ }^{\mathrm{TM}}$ (Biomeda, Foster City, CA, USA), and images were obtained using a laser-scanning confocal microscope (LSM710; Carl Zeiss, Oberkochen, Germany) at a magnification of $\mathrm{x} 400$.

Three-dimensional (3D) cultures and immunohistochemical staining. We prepared 3D cultures of cells as described with a few modifications (17). To mimic the in vivo environment, a type I collagen ( $\mathrm{Col} \mathrm{I})$ gel matrix was reconstituted according to the manufacturer's specifications (Nitta Gelatin, Osaka, Japan), and then $250 \mu \mathrm{l}$ of this mixture was plated into $12-\mathrm{mm}$ polycarbonate filter chambers (3.0 $\mu \mathrm{m}$ Millicell; Millipore, Temecula, CA, USA). MDA-MB-231 cells were seeded at a density of 
$2 \times 10^{5}$ cells/chamber and cultured in the submerged state in culture medium for 7 days, and then in the air-liquid interface state for $24 \mathrm{~h}$. During this period of air-liquid interface culture, cells were concurrently treated with or without IM-412. After the 1-day air-liquid interface culture, the 3D cultures were fixed in Carnoy's solution (ethanol:chloroform:acetic acid, 6:3:1) for $30 \mathrm{~min}$ at $4^{\circ} \mathrm{C}$, and then the fixed samples were embedded in paraffin and sectioned $(3 \mu \mathrm{m})$. For staining, sections were deparaffinized and the endogenous peroxidase activity was blocked. Mouse $\alpha$-SMA antibody (1:500) was used as the primary antibody and the sections were developed using the Cap-Plus $^{\mathrm{TM}}$ Detection kit (Invitrogen); after the development step, samples were counterstained with hematoxylin (Dako, Carpinteria, CA, USA). The stained samples were examined under a microscope and photographed at a magnification of $\mathrm{x} 200$.

Modeling of IM-412-FGFR binding. We performed calculations using Discover 2.98/Insight II with CVFF force field as described by Hagler et al (18). The crystal structure of FGFR3 bound to an ATP mimetic molecule (phosphomethylphosphonic acid adenylate ester) was used as the computational model (4K33.pdb). IM-412 was built from the coordinates of an adenine group, as found in the crystal structure using the Builder Module in Insight II. The computational complex model was initially minimized using 1,000 steps of steepest decent and 3,000 steps of conjugated gradient with a $14.0 \AA$ non-bonded cut-off distance.

Data analysis. Data are represented as means \pm SD. Statistical comparisons between groups were performed using the Student's t-test. Data were considered statistically significant at $\mathrm{P}<0.05, \mathrm{P}<0.01$ or $\mathrm{P}<0.001$ as indicated in the figure legends.

\section{Results}

IM-412 inhibits the invasion and migration in MDA-MB231 cells. TGF- $\beta$ is widely recognized to exhibit pleiotropic activity in biological processes ranging from development, proliferation, differentiation, angiogenesis and immune regulation to tumor biology. Moreover, TGF- $\beta$ signaling plays a prominent role in EMT $(1,3,19)$. Since our previous results demonstrated that the novel compound IM-412 effectively inhibited TGF- $\beta$ signaling in human lung fibroblasts (16), we aimed to investigate whether IM-412 prevents the migration and invasion of cancer cells. First, we examined the cytotoxic effect of IM-412 on MDA-MB-231 cells, which are highly metastatic human breast carcinoma cells. At concentrations above $2 \mu \mathrm{M}$, IM-412 markedly reduced the viability of the cells, but at concentrations below $2 \mu \mathrm{M}$, the viability levels were the same as those in untreated control cells (Fig. 1A). Next, the changes in cell motility induced by IM-412 were investigated using the wound migration (Fig. 1B) and the Transwell invasion assays (Fig. 1C) under comparatively less cytotoxic conditions. IM-412 drastically reduced the abilities of migration and invasion of MDA-MB-231 cells in a dosedependent manner.

IM-412 suppresses the expression of EMT-associated markers in MDA-MB-231 cells. To evaluate the capacity of IM-412 to affect EMT-related markers during MDA-MB-231 cell invasion, we determined the expression levels of EMT-associated molecules by performing western blot analysis (Fig. 2A). IM-412 markedly reduced the expression of mesenchymal markers such as fibronectin, collagen type 1 A 2 and $\alpha$-SMA (ACTA2; $\alpha$-smooth muscle actin). The expression level of $\mathrm{N}$-cadherin was also decreased following IM-412 treatment, but increased expression of E-cadherin, an epithelial marker, was not detected in the MDA-MB-231 cells, which were previously reported to be E-cadherin-negative cells (20). The expression of vimentin, another well-known EMT marker, also remained unchanged in response to IM-412. In close agreement with these results, the expression of EMT-activating transcription factors such as SNAIL, SLUG and ZEB1 was drastically suppressed by IM-412 in a dose-dependent manner.

We also verified the effect of IM-412 on the downregulation of the representative molecule $\alpha$-SMA by performing immunocytochemical and immunohistochemical staining. At $2 \mu \mathrm{M}$, IM-412 significantly downregulated the cellular expression of $\alpha$-SMA (Fig. 2B). Immunohistochemical analysis of 3D collagen-gel-cultured MDA-MB-231 cells revealed numerous invaded cells in the collagen matrix. However, the cells treated with IM-412 did not exhibit invasive activity and expressed $\alpha$-SMA at a low level (Fig. 2C). These results indicated that IM-412 effectively suppresses cell motility by inhibiting the expression of EMT-associated transcription factors and mesenchymal markers.

IM-412 inhibits Smad and non-Smad signaling pathways. To investigate the molecular mechanism underlying IM-412 inhibition of cancer-cell invasion, we first examined the major TGF- $\beta$ downstream signaling pathway, the Smad-dependent pathway. As expected, IM-412 significantly suppressed the phosphorylation of both Smad2 and Smad3 in a dose-dependent manner (Fig. 3A). Since the non-canonical signaling pathway of TGF- $\beta$ has also been clearly shown to fully activate the EMT process (1-3), we examined how non-Smad signaling was affected by IM-412; the phosphorylation levels of p38MAPK, Akt and JNK were significantly decreased by IM-412 in a concentration-dependent manner, but the level of ERK phosphorylation was not affected (Fig. 3B). To determine whether IM-412 influences the upstream molecules of the TGF- $\beta$ signaling pathways, we examined the expression of T $\beta$ RII and T $\beta$ RI. In contrast to our previous findings in lung fibroblasts, IM-412 did not alter the expression of T $\beta$ RII and T $\beta$ RI (Fig. 3C).

IM-412 inhibits FGFR3 phosphorylation. In addition to TGF- $\beta$-family proteins, several other growth factors can induce partial or full EMT by activating receptor tyrosine kinases and triggering downstream signaling cascades $(2,6)$; among the receptor tyrosine kinases, FGFRs, in particular, are involved in diverse cellular processes, including metastasis and EMT (10). In our unpublished data, we demonstrating that another derivative compound of IM-412 [one fluoryl and one methyl group was added to IM-412, 3-(2-chloro-6-fluorobenzyl)-1,6,7-trimethyl-1H-imidazo[2,1-f]purine-2,4(3H,8H)-dione] exhibited the highest inhibitory activity on FGFR3 among 65 kinases and 2-fold higher inhibitory activity against FGFR3 compared with FGFR1 in an in vitro kinase assay. Therefore, we focused 
B

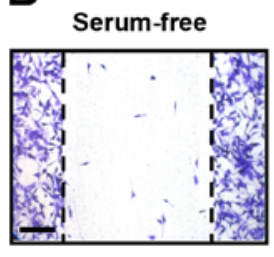

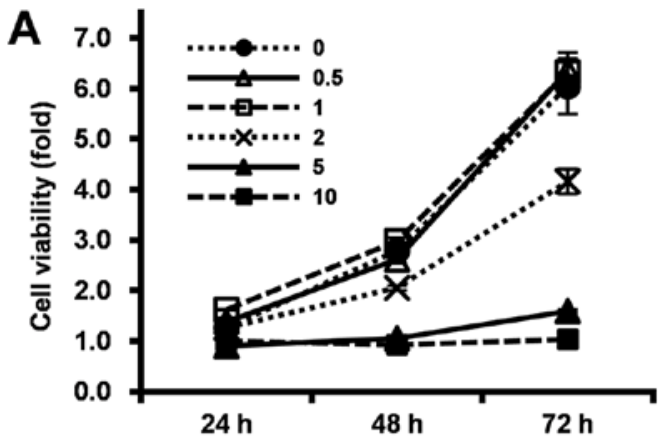
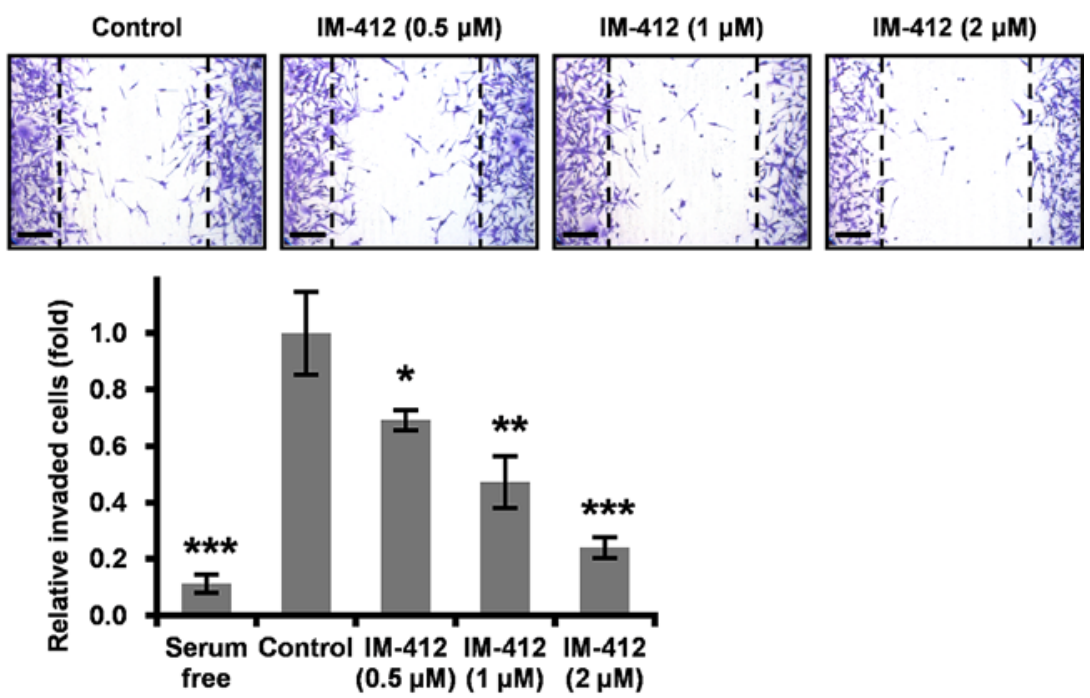

C Serum-free

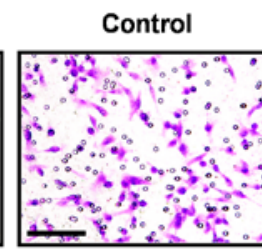

IM-412 (0.5 $\mu \mathrm{M})$
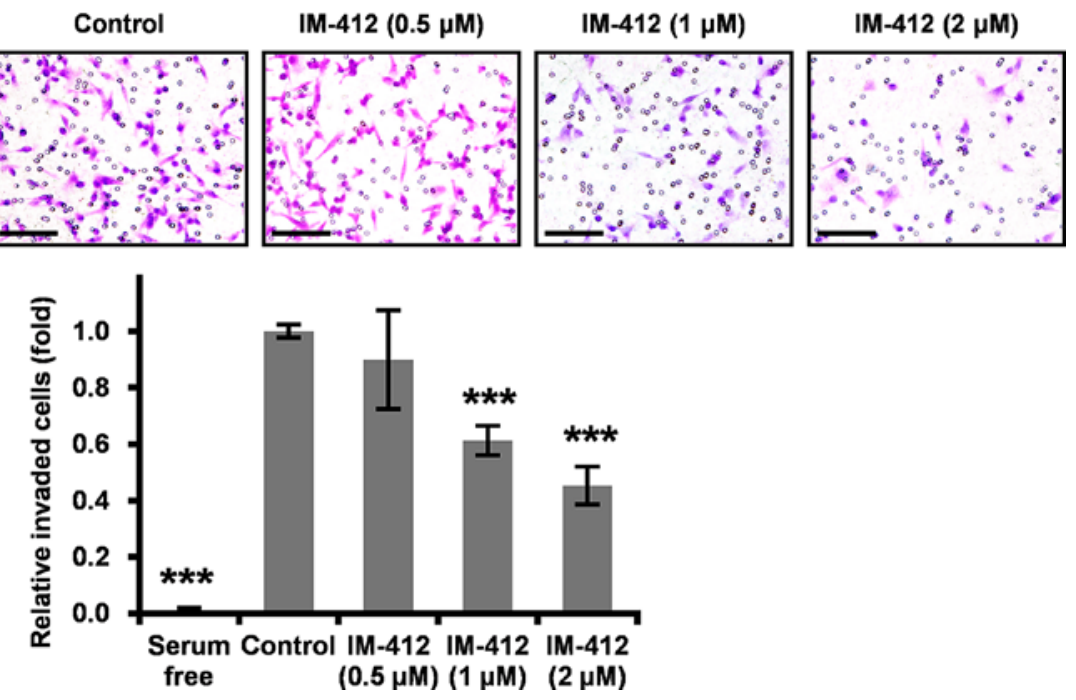

Figure 1. IM-412 inhibits the migration and invasion of MDA-MB-231 cells. (A) MTT assays were performed to examine cell viability. MDA-MB-231 cells were seeded at $8 \times 10^{3}$ cells/well into 24 -well plates and treated with the indicated concentrations of IM-412 for 24,48 or 72 h. Each sample was assayed in triplicate, and the experiment was repeated thrice. (B) To measure the effect of IM-412 on cell migration, MDA-MB-231 cells were seeded at 5x105 cells/well into 6-well plates. The cells were scratched using a 200- $\mu 1$ pipette tip and treated with the indicated concentrations of IM-412 for 24 h, after which the cells were stained with crystal violet and photographed under a microscope at a magnification of $x 40$. Scale bar, $100 \mu \mathrm{m}$. Results were quantified by counting the migrated cells in each experiment. Data shown are means $\pm \mathrm{SD}$ of three independent experiments performed in triplicate; ${ }^{*} \mathrm{P}<0.05 \mathrm{vs}$. control, ${ }^{* *} \mathrm{P}<0.01$ vs. control, ${ }^{* * *} \mathrm{P}<0.001$ vs. control. (C) To examine the IM-412-induced invasiveness of cells, the Transwell invasion assay was performed. MDA-MB-231 cells were seeded at $5 \times 10^{4}$ cells/well in the Matrigel-coated upper chamber. For visualization, the cells were stained with crystal violet and photographed under a microscope at x40 magnification. Scale bar, $100 \mu \mathrm{m}$. Results were quantified by counting the invaded cells in 5 randomly selected regions in each experiment. Data shown are means $\pm \mathrm{SD}$ of three independent experiments performed in triplicate; ${ }^{* * *} \mathrm{P}<0.001$ vs. control.

on FGFR3 as it appeared to be the most probable target molecule of IM-412. We employed computational prediction to identify the binding site of FGFR3 for IM-412 (Fig. 4A). The molecular-modeling results obtained for the FGFR3/IM-412 complex showed the two strong hydrogen bonds between the $\mathrm{N}-\mathrm{H}$ imidazo group of IM-412 and the $\mathrm{C}=\mathrm{O}$ backbone of Ala558 (2.0 $\AA)$, the nitrogen of the purine dione group, and the N-H backbone of Ala558 (2.14 $\AA$ ) at the hinge backbone of 

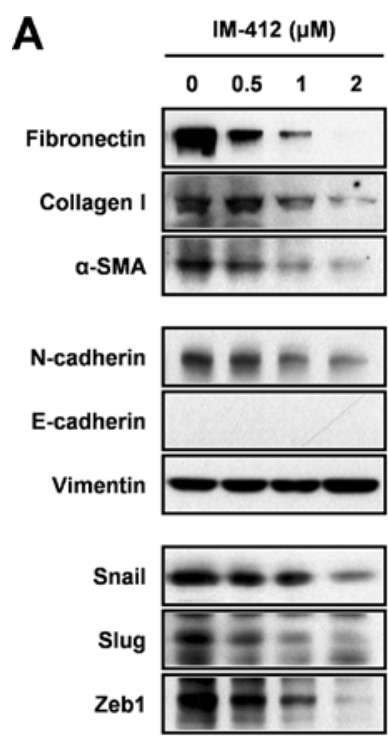

B
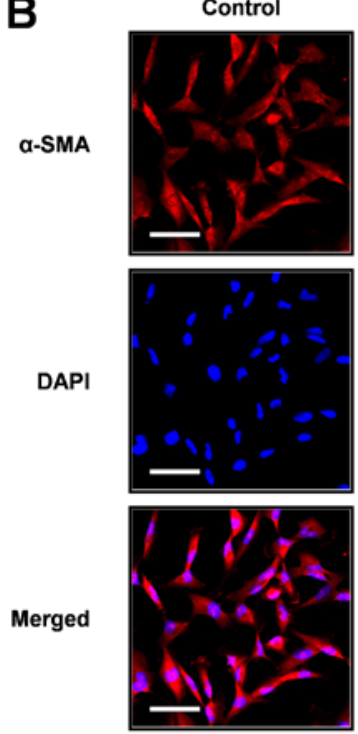

IM-412 (2 $\mu \mathrm{M})$
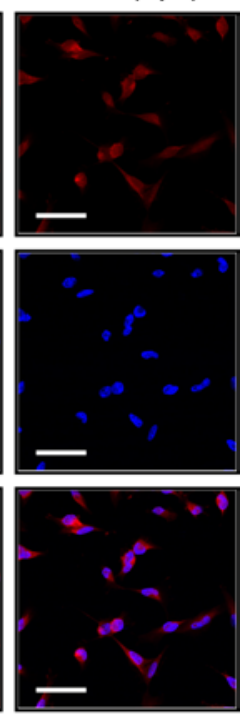

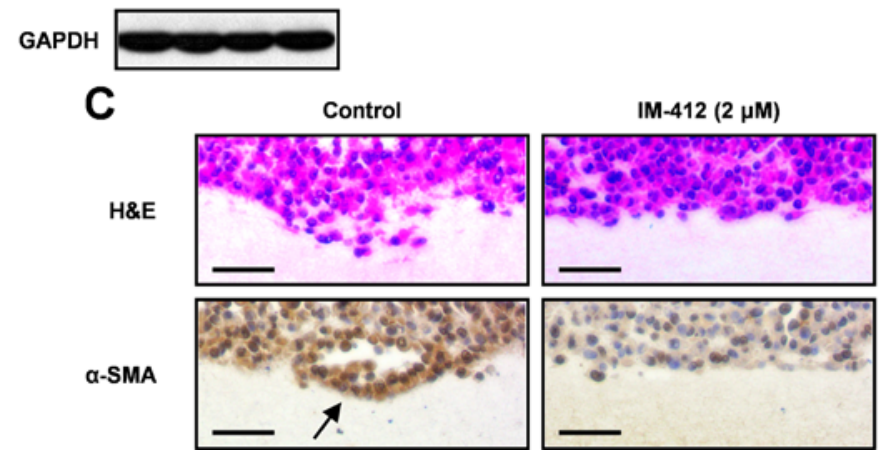

Figure 2. IM-412 suppresses the expression of EMT-associated molecules. (A) MDA-MB-231 cells were treated with the indicated dose of IM- 412 for $24 \mathrm{~h}$, and then total protein was isolated and examined by means of western blotting. The indicated antibodies were used for determining the IM-412-induced levels of EMT markers, EMT-activating transcription factors and mesenchymal markers. (B) MDA-MB-231 cells were seeded on glass coverslips and exposed to the indicated conditions for $24 \mathrm{~h}$. The expression level of $\alpha$-SMA was analyzed by means of immunocytochemical staining performed using an $\alpha$-SMA-specific antibody and Alexa 546-conjugated secondary antibody. Nuclei were stained with DAPI (300 ng/ml). Scale bar, $50 \mu$ m. (C) Immunohistochemical staining pattern of $\alpha$-SMA in the 3D collagen-gel-matrix culture system. MDA-MB-231 cells were seeded on a collagen-loaded chamber and incubated for 7 days, and then treated with IM-412 for $24 \mathrm{~h}$. The arrow indicates invaded cells. Scale bar, $50 \mu \mathrm{m}$.
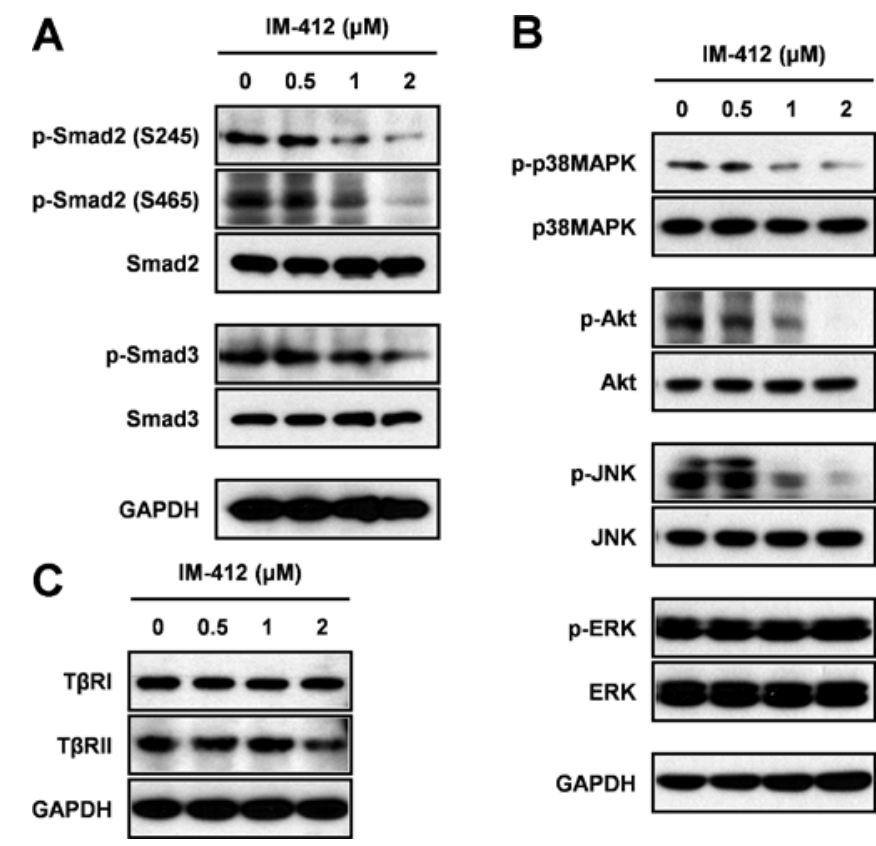

Figure 3. IM-412 inhibits both Smad and non-Smad signaling pathways. MDA-MB-231 cells were treated with the indicated dose of IM- 412 for $24 \mathrm{~h}$, and then total protein was isolated and subjected to western blot analysis. The indicated antibodies were used to examine IM-412-dependent inhibition of (A) Smad signaling; (B) non-Smad signaling; and (C) T $\beta R I$ and T $\beta R I I$ expression. 


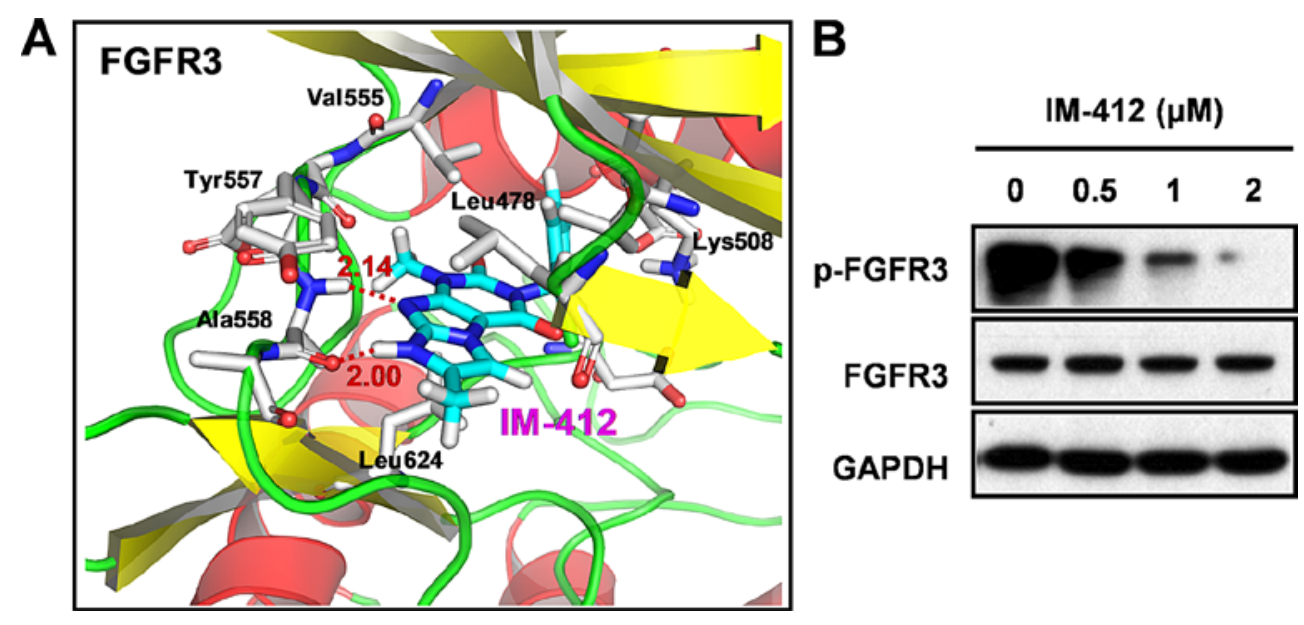

Figure 4. IM-412 suppresses the phosphorylation of FGFR3. (A) Structural model of the FGFR3-IM-412 complex; the ATP-binding pocket in the receptor is shown. The structure was generated using PyMOL software (http://www.pymol.org). (B) MDA-MB-231 cells were treated with the indicated dose of IM-412 for $24 \mathrm{~h}$, and then total protein was isolated and subjected to western blotting using the indicated specific antibodies.

FGFR3. In the structure, the 2-chlorobenzyl is directed toward the gatekeeper residue Val555. Hydrophobic interactions were observed between the benzyl ring and the gatekeeper residue Val555 in addition to the several hydrophobic interactions observed with the 2-chloro substitutions (Lys508 and Ala634). Thus, we considered the imidazopurine structure of IM-412 to be highly active in blocking the ATP-binding-pocket region of FGFR3.

To confirm the potential ability of IM-412 to block the activation of FGFR3, we examined the levels of FGFR3 and phosphorylated FGFR3. The phosphorylation of FGFR3 was significantly inhibited by IM-412, while the expression of FGFR3 protein was not changed (Fig. 4B).

\section{Discussion}

From a cell-based screening of 8,000 small compounds performed using a p3TP-Lux reporter-assay system, IM-412 was isolated as a new inhibitor of TGF- $\beta$ responses (16). Since the TGF- $\beta$ signaling pathway is strongly associated with EMT, we sought to investigate the effect of IM-412 on the EMT pathway in aggressive human breast cancer cells.

MDA-MD-231 is a representative cell line of triple negative breast cancer (TNBC), which lacks the expression of estrogen receptor (ER), progesterone receptor (PR) and human EGF receptor 2 (HER2) (and is therefore commonly referred to as ER-/PR-/HER2-) (21). TNBC is widely recognized for its aggressive phenotype and poor prognosis, and thus researchers are attempting to identify novel targets and develop inhibitors for them as new treatment options for TNBC patients (22). Recently, inhibitors of androgen, EGF and VEGF receptors, FGFR, PARP and tyrosine kinases have been actively studied for use in targeted cancer therapy, and some of these inhibitors are currently being used $(22,23)$. However, the occurrence of resistance to targeted drugs has emerged as a major challenge in the clinic and has highlighted the necessity for developing a novel class of anticancer drugs (24-27).

In the present study, IM-412 potently inhibited the migration and invasion of MDA-MB-231 cells. Although IM-412 did not alter the expression of vimentin and E-cadherin (which are typical EMT markers) relative to their expression in untreated control cells, the compound effectively suppressed the expression of EMT-activating transcription factors (SNAIL, SLUG and ZEB1) and mesenchymal makers (N-cadherin, fibronectin, collagen type 1 A 2 and $\alpha$-SMA). Furthermore, IM-412 treatment led to a reduction in Smad2/3 phosphorylation and also p38MAPK, Akt and JNK phosphorylation, which indicates that IM-412 can block both canonical and non-canonical TGF- $\beta$ signaling pathways. However, the expression of T $\beta$ RII and T $\beta$ RI was not suppressed by IM-412. We also observed that the phosphorylation of T $\beta$ RII was not changed with or without IM-412 (data not shown). Similarly, our previous study showed that the levels of T $\beta$ RI and T $\beta$ RII were not changed by IM-412 in human lung fibroblast, but IM-412 inhibited TGF-mediated T $\beta$ RI and T $\beta$ RII upregulation (16). These findings suggest that the EMT-related signaling molecules that are in common may function distinctly depending on the cell type and on the stimuli and the existing conditions. Although TGF- $\beta$ receptors signal directly upstream of $S m a d 2 / 3$ in the EMT signaling pathway, other kinases such as p38MAPK, Akt, ERK and JNK can also activate Smad signaling through the phosphorylation of the Smad2 linker region (Ser245/250/255), which is induced by various growth-factor receptors, including FGFR $(28,29)$. Moreover, stimulation of these kinases can lead to the induction of EMT-activating transcription factors through the Smad-independent signaling cascade $(2,8)$.

On the basis of the results of computational prediction, we verified that IM-412 effectively inhibited the phosphorylation of FGFR3, but did not affect the receptor's protein level per se. In addition, IM-412 treatment also slightly reduced the phosphorylation of FGFR1 which share a conserved structure and a high level of homology with FGFR3 (data not shown). Given the results of above-mentioned in vitro kinase assay that most of kinases, such as AKT, p38MAPK, ERK, JNK, Src, RAF, RAS, JAK and so on, were not influenced by IM-412 derivative, we carefully considered that IM-412 may not be a general kinase inhibitor.

FGFRs are well-characterized EMT-inducing growthfactor receptors (13) that are frequently overexpressed in cancer cells and are associated with its aggressive 
phenotype $(14,30,31)$. The FGFR-family proteins contain a common extracellular immunoglobulin-like domain and a cytoplasmic tyrosine kinase domain. Phosphorylated FGFR activates phospholipase $\mathrm{C}-\boldsymbol{\gamma}$ and FGFR substrate 2 , and this results in the activation of downstream signaling pathways such as the PKC, STAT and MAPK signaling pathways $(14,15,31)$. Thus, the use of targeted therapies against FGFRs, such as those involving an FGFR1-IIIc-specific antibody (IMC-A1) and FGFR3-specific antibodies (PRO-001 and R3Mab), is considered a new approach for treating cancer patients (11). Moreover, the FGFR1 inhibitor PD173074 has been shown to prevent EMT in head and neck squamous-cell carcinoma (32). In the present study, one limitation was that we did not obtain direct evidence of crosstalk between FGFR/MAPK signaling and Smad cascades, and thus further investigation must be conducted to clarify this potential connection between the signaling pathways.

Collectively, our results demonstrated that the newly identified small molecule IM-412 inhibited the migration and invasion of MDA-MB-231 cells by suppressing FGFR3 signaling. We suggest that further preclinical evaluation of this compound may facilitate the development of a novel therapeutic agent for treating malignant cancers.

\section{Acknowledgements}

The present study was supported by the Nuclear Research and Development Program (2012M2A2A7010422), and in part by the Basic Science Research Program (2013R1A1A2012886) through the National Research Foundation of Korea (NRF) grant funded by the Korean government (MSIP).

\section{References}

1. Derynck R, Muthusamy BP and Saeteurn KY: Signaling pathway cooperation in TGF- $\beta$-induced epithelial-mesenchymal transition. Curr Opin Cell Biol 31: 56-66, 2014.

2. Kalluri R and Weinberg RA: The basics of epithelial-mesenchymal transition. J Clin Invest 119: 1420-1428, 2009.

3. Xu J, Lamouille $\mathrm{S}$ and Derynck R: TGF-beta-induced epithelial to mesenchymal transition. Cell Res 19: 156-172, 2009.

4. Fan YL, Zheng M, Tang YL and Liang XH: A new perspective of vasculogenic mimicry: EMT and cancer stem cells (Review) Oncol Lett 6: 1174-1180, 2013.

5. Kurrey NK, Jalgaonkar SP, Joglekar AV, Ghanate AD, Chaskar PD, Doiphode RY and Bapat SA: Snail and slug mediate radioresistance and chemoresistance by antagonizing p53-mediated apoptosis and acquiring a stem-like phenotype in ovarian cancer cells. Stem Cells 27: 2059-2068, 2009.

6. Lee JK, Joo KM, Lee J, Yoon Y and Nam DH: Targeting the epithelial to mesenchymal transition in glioblastoma: The emerging role of MET signaling. Onco Targets Ther 7: 1933-1944, 2014.

7. Kotiyal S and Bhattacharya S: Breast cancer stem cells, EMT and therapeutic targets. Biochem Biophys Res Commun 453: 112-116, 2014.

8. Tania M, Khan MA and Fu J: Epithelial to mesenchymal transition inducing transcription factors and metastatic cancer. Tumour Biol 35: 7335-7342, 2014

9. Massagué J: TGF $\beta$ signalling in context. Nat Rev Mol Cell Biol 13: 616-630, 2012.

10. Katoh M and Nakagama H: FGF receptors: Cancer biology and therapeutics. Med Res Rev 34: 280-300, 2014.
11. Turner $\mathrm{N}$ and Grose R: Fibroblast growth factor signalling: From development to cancer. Nat Rev Cancer 10: 116-129, 2010.

12. Brooks AN, Kilgour E and Smith PD: Molecular pathways: fibroblast growth factor signaling: a new therapeutic opportunity in cancer. Clin Cancer Res 18: 1855-1862, 2012.

13. Acevedo VD, Gangula RD, Freeman KW, Li R, Zhang Y, Wang F, Ayala GE, Peterson LE, Ittmann M and Spencer DM: Inducible FGFR-1 activation leads to irreversible prostate adenocarcinoma and an epithelial-to-mesenchymal transition. Cancer Cell 12: 559-571, 2007.

14. Feng S, Zhou L, Nice EC and Huang C: Fibroblast growth factor receptors: Multifactorial-contributors to tumor initiation and progression. Histol Histopathol 30: 13-31, 2015.

15. Haugsten EM, Wiedlocha A, Olsnes S and Wesche J: Roles of fibroblast growth factor receptors in carcinogenesis. Mol Cancer Res 8: 1439-1452, 2010

16. Park S, Ahn JY, Lim MJ, Kim MH, Lee SL, Yun YS, Jeong G and Song JY: IM-412 inhibits transforming growth factor betainduced fibroblast differentiation in human lung fibroblast cells. Biochem Biophys Res Commun 399: 268-273, 2010.

17. Yi JY, Hur KC, Lee E, Jin YJ, Arteaga CL and Son YS: TGFbeta1mediated epithelial to mesenchymal transition is accompanied by invasion in the SiHa cell line. Eur J Cell Biol 81: 457-468, 2002.

18. Hagler AT, Lifson S and Dauber P: Consistent force field studies of intermolecular forces in hydrogen-bonded crystals. 2. A benchmark for the objective comparison of alternative force fields. J Am Chem Soc 101: 5122-5130, 1979.

19. Lamouille S, Xu J and Derynck R: Molecular mechanisms of epithelial-mesenchymal transition. Nat Rev Mol Cell Biol 15: 178-196, 2014.

20. Cailleau R, Young R, Olivé M and Reeves WJ Jr: Breast tumor cell lines from pleural effusions. J Natl Cancer Inst 53: 661-674, 1974.

21. Kao J, Salari K, Bocanegra M, Choi YL, Girard L, Gandhi J, Kwei KA, Hernandez-Boussard T, Wang P, Gazdar AF, et al: Molecular profiling of breast cancer cell lines defines relevant tumor models and provides a resource for cancer gene discovery. PLoS One 4: e6146, 2009.

22. Schmadeka R, Harmon BE and Singh M: Triple-negative breast carcinoma: Current and emerging concepts. Am J Clin Pathol 141: 462-477, 2014.

23. Yadav BS, Sharma SC, Chanana P and Jhamb S: Systemic treatment strategies for triple-negative breast cancer. World J Clin Oncol 5: 125-133, 2014.

24. Ciardiello F and Tortora G: EGFR antagonists in cancer treatment. N Engl J Med 358: 1160-1174, 2008.

25. Ellis LM and Hicklin DJ: VEGF-targeted therapy: Mechanisms of anti-tumour activity. Nat Rev Cancer 8: 579-591, 2008.

26. Grose R and Dickson C: Fibroblast growth factor signaling in tumorigenesis. Cytokine Growth Factor Rev 16: 179-186, 2005.

27. Takeuchi K and Ito F: Receptor tyrosine kinases and targeted cancer therapeutics. Biol Pharm Bull 34: 1774-1780, 2011.

28. Hough C, Radu M and Doré JJ: Tgf-beta induced Erk phosphorylation of smad linker region regulates smad signaling. PLoS One 7: e42513, 2012.

29. Kamato D, Burch ML, Piva TJ, Rezaei HB, Rostam MA, Xu S, Zheng W, Little PJ and Osman N: Transforming growth factor- $\beta$ signalling: Role and consequences of Smad linker region phosphorylation. Cell Signal 25: 2017-2024, 2013.

30. Wendt MK, Taylor MA, Schiemann BJ, Sossey-Alaoui K and Schiemann WP: Fibroblast growth factor receptor splice variants are stable markers of oncogenic transforming growth factor $\beta 1$ signaling in metastatic breast cancers. Breast Cancer Res 16: R24, 2014.

31. Tiong KH, Mah LY and Leong C-O: Functional roles of fibroblast growth factor receptors (FGFRs) signaling in human cancers. Apoptosis 18: 1447-1468, 2013.

32. Nguyen PT, Tsunematsu T, Yanagisawa S, Kudo Y, Miyauchi M, Kamata N and Takata T: The FGFR1 inhibitor PD173074 induces mesenchymal-epithelial transition through the transcription factor AP-1. Br J Cancer 109: 2248-2258, 2013. 